\title{
Different dosage regimens of Eptinezumab for the treatment of migraine: a meta- analysis from randomized controlled trials
}

Zeya Yan ${ }^{1 \dagger}$, Tao Xue ${ }^{1 \dagger}$, Shujun Chen², Xin Wu' ${ }^{1}$ Xingyu Yang ${ }^{1}$, Guangjie Liu', Shan Gao ${ }^{3}$, Zhouqing Chen ${ }^{1 *}$ and Zhong Wang ${ }^{1 *}$ (D)

\begin{abstract}
Background: Migraine is one of the most common neurological diseases around the world and calcitonin generelated peptide (CGRP) plays an important role in its pathophysiology. Therefore, in the present study, we evaluated the efficacy of monoclonal antibodies blocking the CGRP ligand or receptor in episodic and chronic migraine.

Objective: The objective of our study is implementing a meta-analysis to systematically evaluate the efficacy and safety of eptinezumab for the treatment of migraine compared with placebo.

Method: We searched the Medline, Embase, Cochrane Library and Clinicaltrials.gov for randomized controlled trials (RCTs) which were performed to evaluate eptinezumab versus placebo for migraine up to September 2020. The data was assessed by Review Manager 5.3 software. The risk ratio (RR) and standard mean difference (SMD) were analyzed using dichotomous outcomes and continuous outcomes respectively with a random effect model.
\end{abstract}

Result: We collected 2739 patients from 4 RCTs: the primary endpoint of efficacy was the change from baseline to week 12 in mean monthly migraine days (MMDs). We found that eptinezumab (30 mg, $100 \mathrm{mg}, 300 \mathrm{mg}$ ) led to a significant reduction in MMDs $(P=0.0001, P<0.00001, P<0.00001)$ during 12 weeks compared with placebo, especially with $300 \mathrm{mg}$. For the safety, we compared and concluded the treatment emergent adverse events (TEAEs) of the 4 RCTs. This indicated no evident statistical difference between eptinezumab and placebo.

Conclusions: In the present study, we found that eptinezumab is safe and has significant efficacy in the treatment of migraine, especially the dose of $300 \mathrm{mg}$.

Keywords: Eptinezumab, Migraine, Dosage regimen, Meta-analysis

\section{Introduction}

Migraine is one of the most prevalent disorders in neurologic field which can be characterized by attacks of headache, hypersensitivity to sound and light stimulation, nausea and vomiting [1]. Commonly, migraine can

\footnotetext{
*Correspondence: zqchen6@163.com; wangzhong761@163.com

'Zeya Yan and Tao Xue contributed equally to this work.

'Department of Neurosurgery \& Brain and Nerve Research Laboratory, The First Affiliated Hospital of Soochow University, 188 Shizi Street, Suzhou 215006, Jiangsu Province, China

Full list of author information is available at the end of the article
}

be divided into episodic migraine and chronic migraine. Episodic migraine is defined as headache which occurs on fewer than 15 days per month, while chronic migraine on 15 or more days per month for at least 3 months or having the features of migraine at least 8 days per month [2]. Presently, migraine has disturbed the peaceful life of more than $16 \%$ global population [3, 4]. Until now, the primary goals of migraine treatment include reducing headache frequency, relieving pain and restoring function. Broadly, improvement of physical and psychological health and some other therapies such

(c) The Author(s). 2021 Open Access This article is licensed under a Creative Commons Attribution 4.0 International License, which permits use, sharing, adaptation, distribution and reproduction in any medium or format, as long as you give appropriate credit to the original author(s) and the source, provide a link to the Creative Commons licence, and indicate if changes were made. The images or other third party material in this article are included in the article's Creative Commons licence, unless indicated otherwise in a credit line to the material. If material is not included in the article's Creative Commons licence and your intended use is not permitted by statutory regulation or exceeds the permitted use, you will need to obtain permission directly from the copyright holder. To view a copy of this licence, visit http://creativecommons.org/licenses/by/4.0/ The Creative Commons Public Domain Dedication waiver (http://creativecommons.org/publicdomain/zero/1.0/) applies to the data made available in this article, unless otherwise stated in a credit line to the data. 


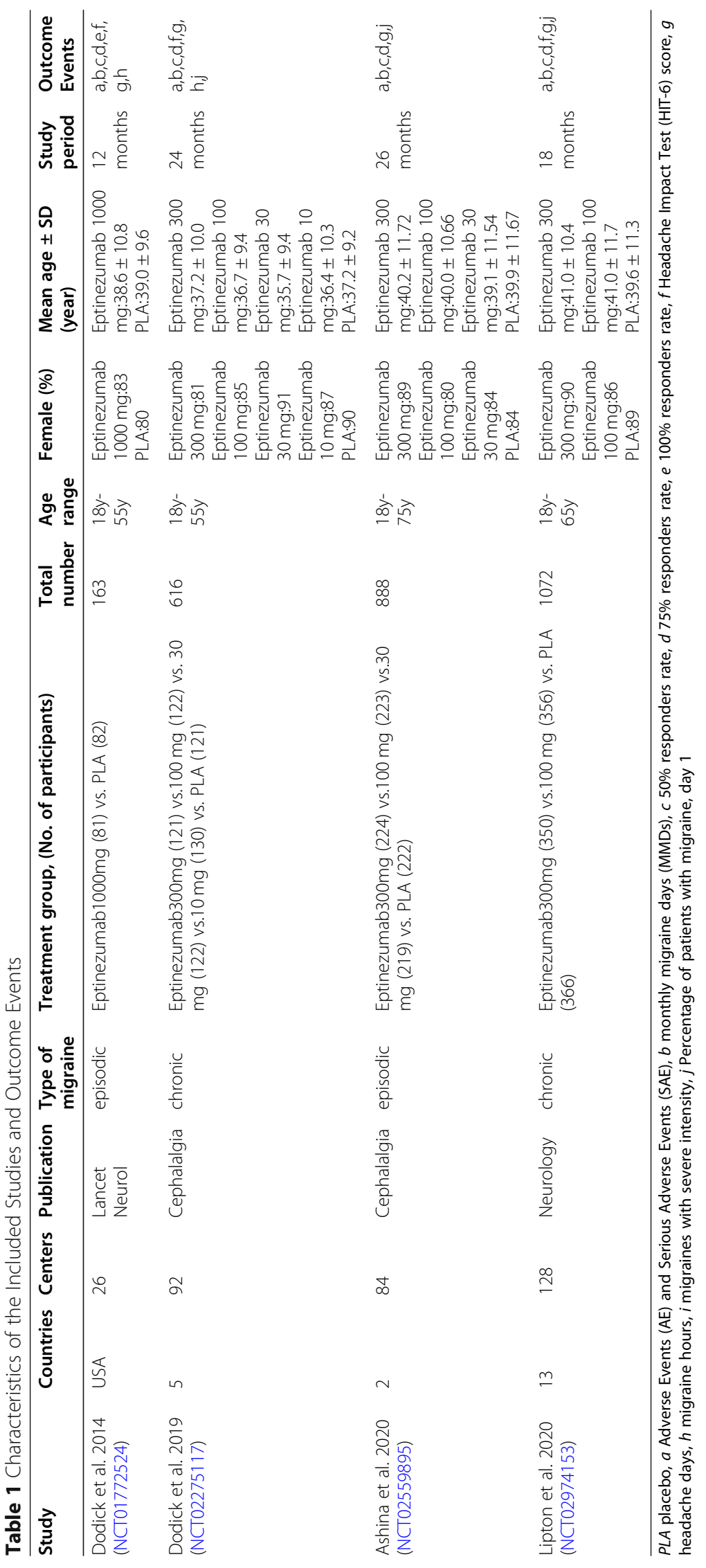


as pharmacotherapy which will be mainly discussed in the following part are included in treatment strategies [5].

Patients with a few migraine attacks per month can be managed with effective acute therapy. For some patients, nonpharmacologic intervention such as taking a break is enough to relieve the symptom of migraine, while the others need some nonspecific analgesics such as NSAIDs [6]. If the patients are insensitive to nonspecific analgesics, migrainespecific treatment such as triptans will be a better choice. Triptans, the classical drugs of migraine which were widely used over past decades, include sumatriptan, eletriptan, rizatriptan, almotriptan, zolmitriptan, naratriptan, frovatriptan and so on [7]. Moreover, ergots also can be used for the treatment of migraine, but those drugs have not been recommended because of the poor efficacy and adverse reactions compared with triptans. Generally, these drugs are effective and safe in appropriate dose but they occasionally cause an overuse of acute medication in some patients. In addition, drugs for acute treatment have not exhibited obvious advantages on the preventive treatment for migraine. Therefore, further studies are needed on the prophylactic medication, such as monoclonal antibodies against calcitonin gene-related peptide (CGRP) or its receptor, antihypertensives, anticonvulsants, antidepressants, botulinum toxin (the latter only for chronic migraine) [8, 9].

The release of CGRP plays an important role in migraine pathophysiology, which has been observed after the migraine attack $[10,11]$. Over the past few years, the efficacy of monoclonal antibodies blocking the CGRP ligand or receptor including galcanezunab, fremanezumab, erenumab and ubrogepant have been demonstrated in both episodic and chronic migraine [12]. Eptinezumab (ALD 403), a new monoclonal antibody that selectively inhibits both $\alpha$-CGRP and $\beta$-CGRP, was available in the market since February 2019. However, there were no systematic review or meta-analysis comprehensively

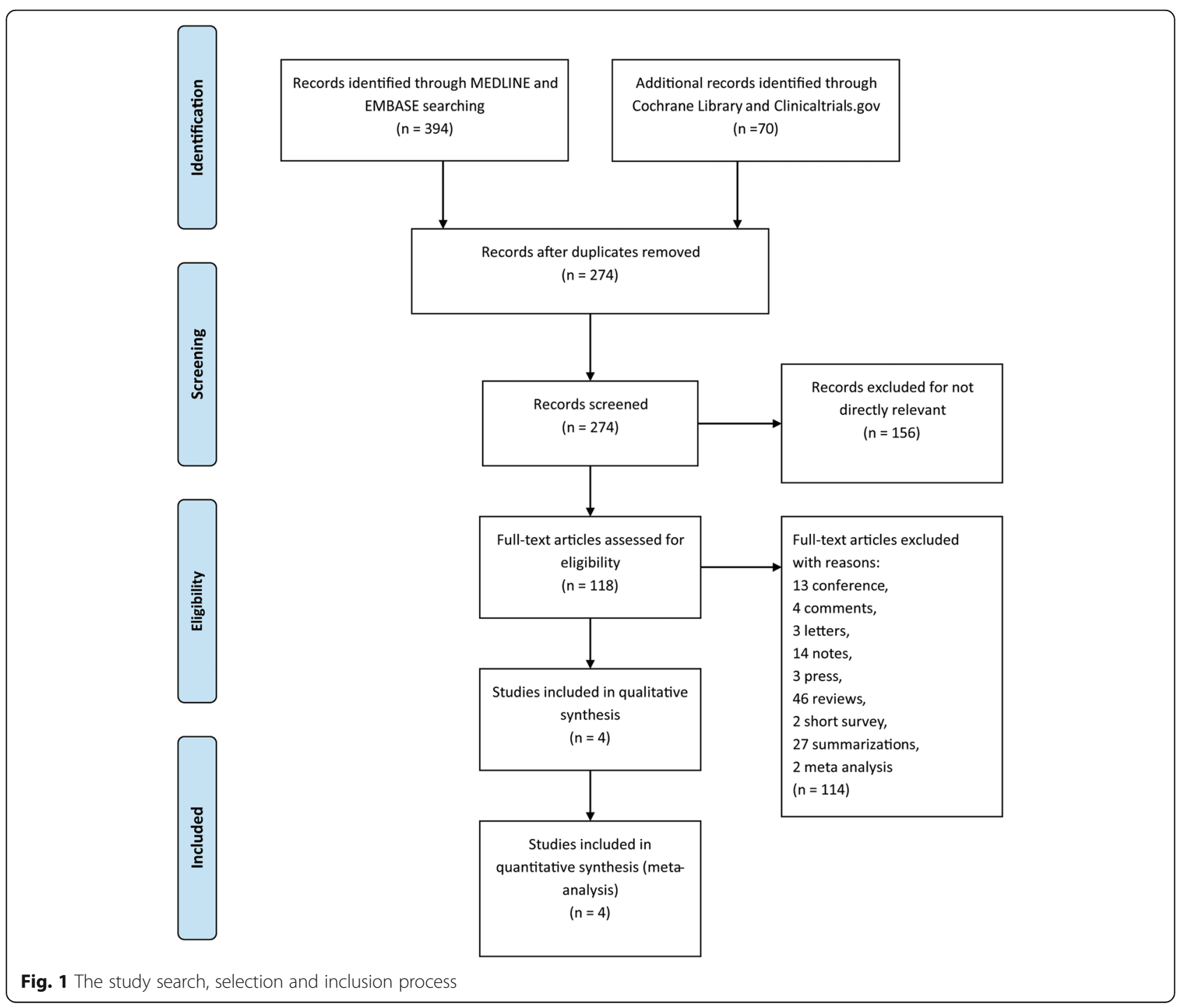


evaluating the efficacy and safety of eptinezumab in the treatment of migraine [13-15].

Therefore, in the present study, we performed a meta-analysis to discuss different dosage regimen of eptinezumab for the treatment of migraine. In the previous clinical trials, eptinezumab had exhibited flexible dosing regimens $(10 \mathrm{mg}, 30 \mathrm{mg}, 100 \mathrm{mg}, 300$ $\mathrm{mg}, 1000 \mathrm{mg}$ ). During our study, we combined different doses of eptinezumab to analyze the efficacy and safety for the therapy of episodic and chronic migraine [16-19].

\section{Methods}

\section{Study protocol}

Before we started the project, we drafted a research protocol by following the Cochrane Collaboration format [20]. The meta-analysis was not registered.

\section{Search strategy}

Original researches in the MEDLINE, Embase, Cochrane Library and Clinicaltrials.gov were searched using the following terms: [("eptinezumab and migraine")
("ALD403 and migraine")] until September 2020. Moreover, to make sure all relevant studies have been included, we screened reference lists of relevant articles manually.

\section{Study selection}

Studies were included as follows: (1) study type was randomized clinical trials; (2) enrolled participants diagnosed with migraine; (3) study used eptinezumab as intervention; (4) study period was over 12 months; (5) participants were over 18 years old. Studies were excluded as follows: (1) types of study: retrospective studies, cohort studies, case reviews and case reports; (2) control: active control (i.e. that a known, effective treatment as opposed to a placebo is compared with an experimental treatment).

\section{Data extraction}

All the data were extracted independently by 2 investigators (ZYY and TX) and any disagreements were settled through discussion. After several selections and assessments, the basic information of the included trails (first

\begin{tabular}{|c|c|c|c|c|c|c|c|c|c|c|c|}
\hline \multirow[b]{2}{*}{ Study or Subgroup } & \multicolumn{3}{|c|}{ Experimental } & \multicolumn{3}{|c|}{ Control } & \multicolumn{3}{|c|}{ Std. Mean Difference } & \multirow{2}{*}{\multicolumn{2}{|c|}{$\begin{array}{l}\text { Std. Mean Difference } \\
\text { IV. Random, } 95 \% \mathrm{Cl}\end{array}$}} \\
\hline & Mean & SD & Total & Mean & SD & Total & Weight & IV, Random, $95 \% \mathrm{Cl}$ & Year & & \\
\hline \multicolumn{12}{|c|}{ 1.1.1 10mg Eptinezumab } \\
\hline $\begin{array}{l}\text { Dodick } 2019 \\
\text { Subtotal }(95 \% \mathrm{Cl})\end{array}$ & -6.7 & 6.9 & $\begin{array}{l}123 \\
123\end{array}$ & -5.5 & 6.82 & $\begin{array}{l}116 \\
116\end{array}$ & $\begin{array}{l}100.0 \% \\
100.0 \%\end{array}$ & $\begin{array}{l}-0.17[-0.43,0.08] \\
-0.17[-0.43,0.08]\end{array}$ & 2019 & & \\
\hline \multicolumn{12}{|c|}{ Heterogeneity: Not applicable } \\
\hline \multicolumn{12}{|c|}{ 1.1.2 30mg Eptinezumab } \\
\hline Dodick 2019 & -7.9 & 6.46 & 117 & -5.5 & 6.82 & 116 & $34.2 \%$ & $-0.36[-0.62,-0.10]$ & 2019 & & \\
\hline $\begin{array}{l}\text { Ashina } 2020 \\
\text { Subtotal }(95 \% \mathrm{Cl})\end{array}$ & -4 & 3.12 & $\begin{array}{l}223 \\
340\end{array}$ & -3.2 & 3.04 & $\begin{array}{l}222 \\
338\end{array}$ & $\begin{array}{r}65.8 \% \\
100.0 \%\end{array}$ & $\begin{array}{l}-0.26[-0.45,-0.07] \\
-0.29[-0.45,-0.14]\end{array}$ & 2020 & & \\
\hline \multicolumn{12}{|c|}{$\begin{array}{l}\text { Heterogeneity: } \mathrm{Tau}^{2}=0.00 ; \mathrm{Chi}^{2}=0.38, \mathrm{df}=1(\mathrm{P}=0.54) ; \mathrm{I}^{2}=0 \% \\
\text { Test for overall effect: } \mathrm{Z}=3.80(\mathrm{P}=0.0001)\end{array}$} \\
\hline \multicolumn{12}{|c|}{ 1.1.3 100mg Eptinezumab } \\
\hline Dodick 2019 & -7.6 & 6.74 & 118 & -5.5 & 6.82 & 116 & $16.7 \%$ & $-0.31[-0.57,-0.05]$ & 2019 & & \\
\hline Ashina 2020 & -3.9 & 2.88 & 221 & -3.2 & 3.04 & 222 & $31.8 \%$ & $-0.24[-0.42,-0.05]$ & 2020 & & \\
\hline Lipton 2020 & -7.7 & 5.83 & 356 & -5.6 & 5.8 & 366 & $51.4 \%$ & $-0.36[-0.51,-0.21]$ & 2020 & & \\
\hline Subtotal $(95 \% \mathrm{Cl})$ & & & 695 & & & 704 & $100.0 \%$ & $-0.31[-0.42,-0.21]$ & & & \\
\hline \multicolumn{12}{|c|}{ Heterogeneity: $\mathrm{Tau}^{2}=0.00 ; \mathrm{Chi}^{2}=1.06, \mathrm{df}=2(\mathrm{P}=0.59) ; \mathrm{I}^{2}=0 \%$} \\
\hline \multicolumn{12}{|c|}{ 1.1.4 300mg Eptinezumab } \\
\hline Dodick 2019 & -8.2 & 6.82 & 114 & -5.5 & 6.82 & 116 & $16.6 \%$ & $-0.39[-0.66,-0.13]$ & 2019 & & \\
\hline Ashina 2020 & -4.3 & 3.05 & 222 & -3.2 & 3.04 & 222 & $32.1 \%$ & $-0.36[-0.55,-0.17]$ & 2020 & & \\
\hline Lipton 2020 & -8.2 & 5.8 & 350 & -5.6 & 5.8 & 366 & $51.3 \%$ & $-0.45[-0.60,-0.30]$ & 2020 & & \\
\hline Subtotal $(95 \% \mathrm{Cl})$ & & & 686 & & & 704 & $100.0 \%$ & $-0.41[-0.52,-0.30]$ & & & \\
\hline \multicolumn{12}{|c|}{ Heterogeneity: $\mathrm{Tau}^{2}=0.00 ; \mathrm{Chi}^{2}=0.53, \mathrm{df}=2(\mathrm{P}=0.77) ; \mathrm{I}^{2}=0 \%$} \\
\hline \multicolumn{12}{|c|}{ 1.1.5 1000mg Eptinezumab } \\
\hline $\begin{array}{l}\text { Dodick } 2014 \\
\text { Subtotal }(95 \% \mathrm{Cl})\end{array}$ & -5.6 & 3 & $\begin{array}{l}78 \\
78\end{array}$ & -4.6 & 3.6 & $\begin{array}{l}80 \\
80\end{array}$ & $\begin{array}{l}100.0 \% \\
100.0 \%\end{array}$ & $\begin{array}{l}-0.30[-0.61,0.01] \\
-0.30[-0.61,0.01]\end{array}$ & 2014 & & \\
\hline \multicolumn{12}{|c|}{$\begin{array}{l}\text { Heterogeneity: Not applicable } \\
\text { Test for overall effect: } Z=1.87(P=0.06)\end{array}$} \\
\hline Test for subgroup diffe & ences: $\mathrm{C}$ & $\mathrm{Chi}^{2}=4$ & 4.01, df & $=4(\mathrm{P}$ & $=0.40$ & ),$I^{2}=0$ & $0.2 \%$ & & & $\begin{array}{ccc}-0.5 & -0.25 & 0 \\
\text { Favours [experimental] }\end{array}$ & 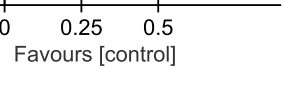 \\
\hline $\begin{array}{l}\text { ig. } 2 \text { The pooled st } \\
\text { iamond indicates th }\end{array}$ & edard $n$ & $\begin{array}{l}\text { nean } \\
\text { ated } S\end{array}$ & $\begin{array}{l}\text { differe } \\
\text { MD wi }\end{array}$ & $\begin{array}{l}\text { nce }(S) \\
\text { ith } 95 \%\end{array}$ & $\begin{array}{l}\text { MD) } \mathrm{C} \\
\% \text { con }\end{array}$ & fidence & $\begin{array}{l}\text { nthly mig } \\
\text { ce interva }\end{array}$ & $\begin{array}{l}\text { graine days (MMDs) ir } \\
\text { al (Cl) for the pooled }\end{array}$ & patie & treatment doses com & npared with placebo, the \\
\hline
\end{tabular}


author, publication, country, centers, and treatment groups), patient characteristics (Age range, mean age and gender), study period and outcome events were used to extract the data (Table 1).

\section{Outcomes}

The primary efficacy outcome is mean monthly migraine days (MMDs), baseline to 12 week. Secondary efficacy endpoint included: patients with a $75 \%$ reduction in migraine days from baseline ( $75 \%$ responder rate), patients with a $50 \%$ reduction in migraine days from baseline (50\% responder rate) and patients with migraine 1 day after dosing, baseline to 12 weeks. In addition, we choose the treatment emergent adverse events (TEAEs) as the safety endpoint.

\section{Summary measures and synthesis of results}

Review manager 5.3 was used to assess the data. Estimated standard mean differences and estimated risk ratio (standard mean difference [SMD] or risk ratio [RR]; 95\% confidence interval $[\mathrm{CI}]$ ) were calculated using a random effects model. The $\mathrm{I}^{2}$ statistic was used to estimate the statistical heterogeneity as follows: $\mathrm{I}^{2}<30 \%$ represents "low heterogeneity," $30 \%<\mathrm{I}^{2}<50 \%$ means "moderate heterogeneity" and $\mathrm{I}^{2}>50 \%$ means "substantial heterogeneity." A $<0.05 P$-value was considered to be significant for all analyses, and tests are two-tailed.

\section{Risk of Bias}

The risk-of-bias plot was assessed using Review Manager 5.3 software (The Cochrane Collaboration, Oxford, UK) for individual studies. The unified standard of the

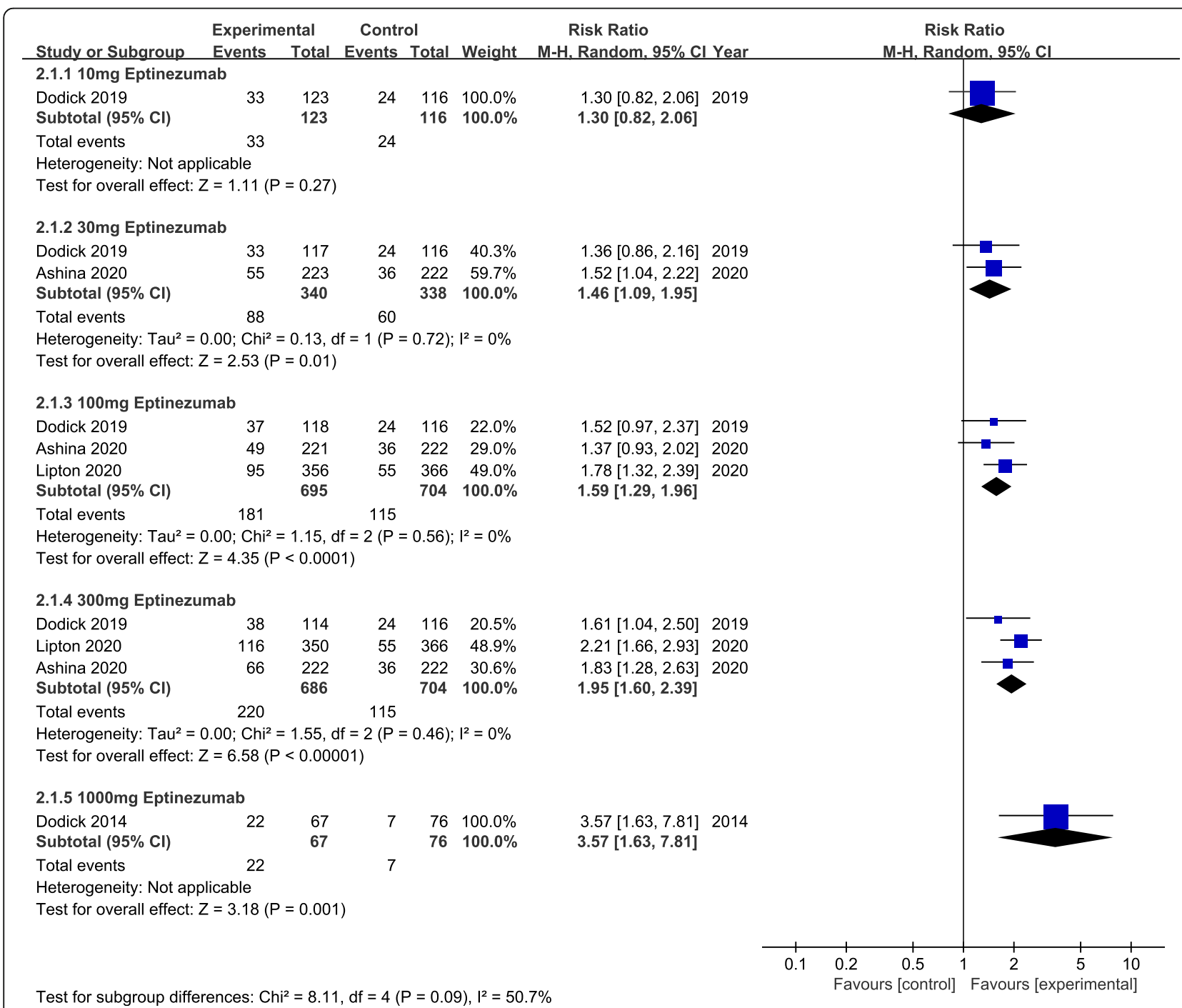

Fig. 3 The pooled risk ratio (RR) of $75 \%$ responder rate (defined as patients with a $75 \%$ reduction in migraine days from baseline) in different treatment doses compared with placebo, the diamond indicates the estimated RR with 95\% confidence interval (Cl) for the pooled patients 
Cochrane Collaboration was applied to assess the risk of bias for RCTs, which included selection bias, performance bias, detection bias, attrition bias, reporting bias, and other potential biases.

\section{Results}

\section{Search results}

A total of 464 researches and abstracts from Medline, Embase, Cochrane library and Clinicaltrials.gov were identified. Among them, 190 studies were excluded due to duplicates. Further, 178 studies were excluded as they were irrelevant, such as research on other drugs or into the etiological analysis of migraine. After removing duplicates and uncorrelated titles, 96 of these articles were directly related to the topic of interest. Among them, 92 full text articles were excluded, which included 13 conferences, 4 comments, 46 reviews, 2 short survey and 27 summarizations. Finally, 4 RCTs containing 2739 patients were included in our meta-analysis. The detailed process of screening is shown in Fig. 1.

\section{Different dosage regimen for the efficacy and safety}

The primary efficacy outcome was mean monthly migraine day (MMDs) (Fig. 2). From the perspective of primary efficacy outcome, treatment with $30 \mathrm{mg}(\mathrm{MD}=-$ 0.29, 95\% CI:-0.45 - 0.14, $P=0.0001), 100 \mathrm{mg}$ (MD = 0.31, 95\% CI:-0.42 - 0.21, $\quad P<0.00001)$ and $300 \mathrm{mg}$ $(\mathrm{MD}=-0.41,95 \% \mathrm{CI}:-0.52 \sim-0.30, P<0.00001)$ eptinezumab showed significant efficacy compared to the placebo. Whereas, for the secondary efficacy endpoint (75\% responder rate, $50 \%$ responder rate and patients with migraine 1 day after dosing) (Figs. 3, 4 and 5), the outcomes were abounded with the MMDs. Initially, for the $75 \%$ responder rate, treatment with $30 \mathrm{mg}(\mathrm{RR}=1.46$,

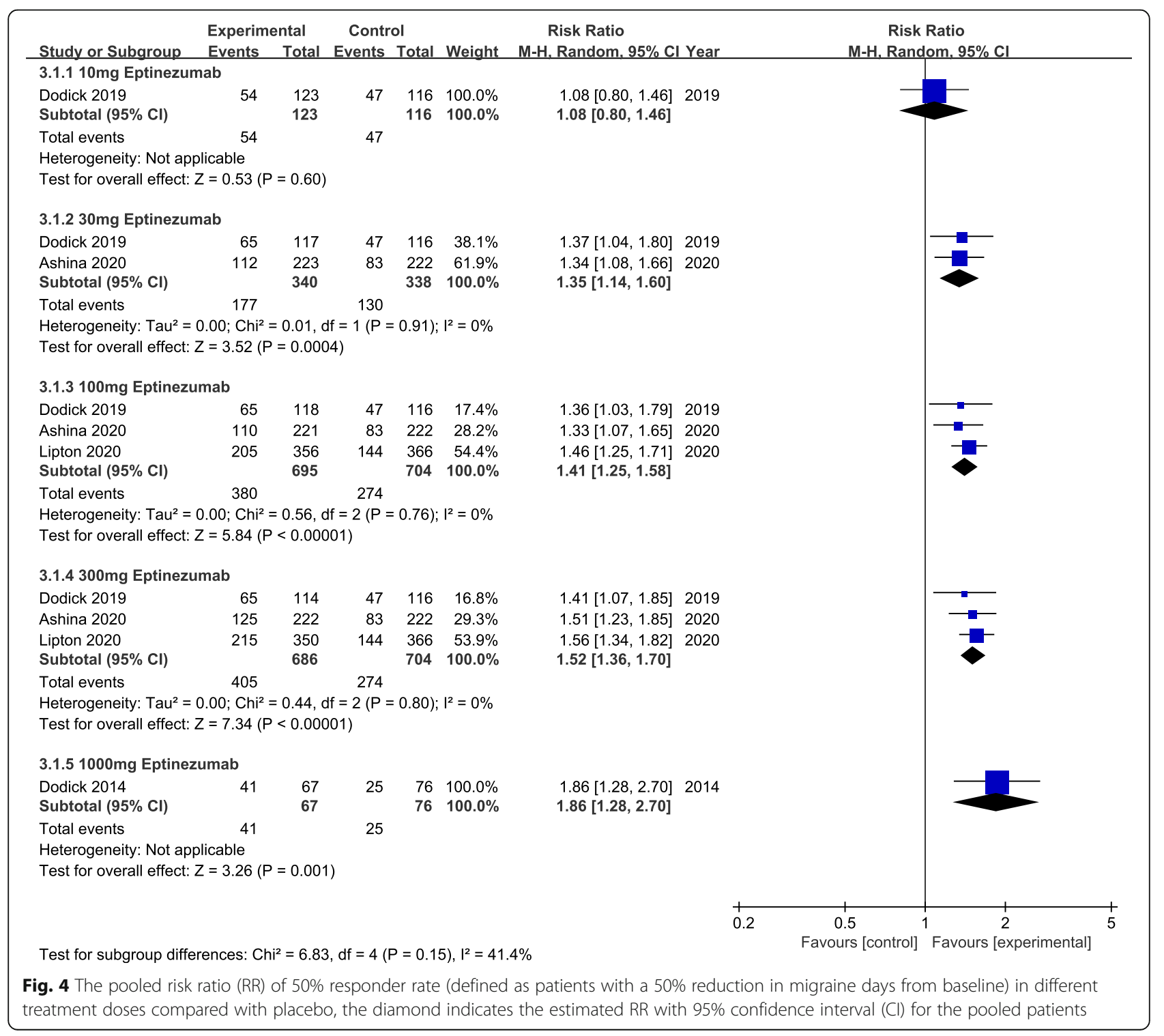




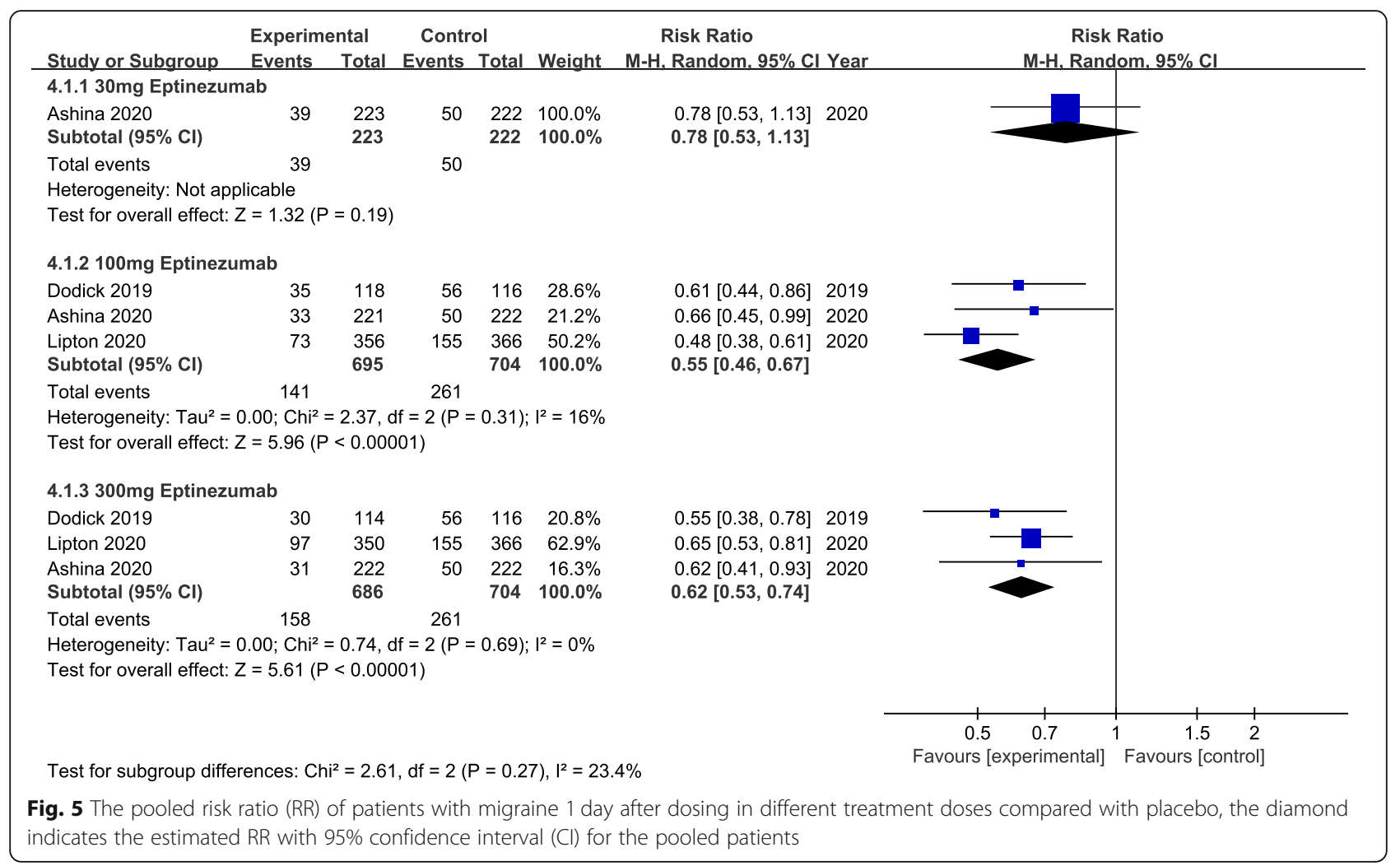

95\% CI:1.09 1.95, $P=0.01), 100 \mathrm{mg}(\mathrm{RR}=1.59,95 \% \mathrm{CI}:$ $1.29 \sim 1.96, P<0.0001), 300 \mathrm{mg}(\mathrm{RR}=1.95,95 \% \mathrm{CI}: 1.60 \sim$ 2.39, $P<0.00001)$ and $1000 \mathrm{mg}(\mathrm{RR}=3.57,95 \% \mathrm{CI}: 1.63$ 7.81, $P=0.001$ ) indicated that they could increase the rate significantly. Further, for the $50 \%$ responder rate, treatment with $30 \mathrm{mg}(\mathrm{RR}=1.35,95 \% \mathrm{CI}: 1.14 \sim 1.60, P=$ $0.0004), \quad 100 \mathrm{mg} \quad(\mathrm{RR}=1.41, \quad 95 \% \quad \mathrm{CI}: 1.25 \sim 1.58, \quad P<$ $0.00001), \quad 300 \mathrm{mg} \quad(\mathrm{RR}=1.52, \quad 95 \% \quad \mathrm{CI}: 1.36 \sim 1.70, \quad P<$ $0.00001)$ and $1000 \mathrm{mg}(\mathrm{RR}=1.86,95 \% \mathrm{CI}: 1.28 \sim 2.70$, $P=0.001)$ eptinezumab also showed improved efficacy compared to the placebo. Nevertheless, from the perspective of the data related to patients with migraine 1 day after dosing, treatment with $100 \mathrm{mg}(\mathrm{RR}=0.55,95 \%$ CI:0.46 0.67, $P<0.00001)$ and $300 \mathrm{mg}(\mathrm{RR}=0.65,95 \%$ CI:0.53 0.74, $P<0.00001)$ appeared to be more effective than $30 \mathrm{mg}(\mathrm{RR}=0.78,95 \% \mathrm{CI}: 0.53 \sim 1.13, P=0.19)$ compared with the placebo.

Of all the 2739 patients receiving eptinezumab, no death occurred during the treatment period. The common adverse effects contained upper respiratory tract infection, nausea and sinus congestion. Therefore, we summarized the adverse events which showed the treatment with $10 \mathrm{mg}(\mathrm{RR}=1.01,95 \% \mathrm{CI}: 0.82 \sim 1.26, p=0.91)$, $30 \mathrm{mg}(\mathrm{RR}=0.92, \quad 95 \% \quad \mathrm{CI}: 0.77 \sim 1.10, \quad p=0.35), 100$ $\operatorname{mg}(\mathrm{RR}=1.01,95 \% \mathrm{CI}: 0.91 \sim 1.11, p=0.92), 300 \mathrm{mg}(\mathrm{RR}=$ 1.06, 95\% CI:0.96 1.17, $p=0.24), 1000 \mathrm{mg} \quad(\mathrm{RR}=1.08$, 95\% CI:0.82 1.43, $p=0.58$ ) had no evident statistical difference between eptinezumab and placebo (Fig. 6).

\section{Dosage regimen of $100 \mathrm{mg}$ vs. $300 \mathrm{mg}$}

Further, study was carried out to compare the efficacy between the $100 \mathrm{mg}$ and $300 \mathrm{mg}$ (Fig. 7a-d). Results from this comparison showed that the treatment with 300 $\operatorname{mg}$ (MMDs, $\mathrm{MD}=0.10,95 \% \mathrm{CI}: 0.00 \sim 0.21, p=0.06 ; 75 \%$ responder rate, $\mathrm{RR}=0.81,95 \% \mathrm{CI}: 0.69 \sim 0.96, p=0.01$; $50 \%$ responder rate, $\mathrm{RR}=0.93,95 \% \mathrm{CI}: 0.85 \sim 1.02, p=$ 0.11 ; patients with migraine 1 day after dosing, $\mathrm{RR}=$ 0.92, 95\% CI:0.69 1.23, $p=0.58$ ) was more promising than $100 \mathrm{mg}$. Meanwhile, as shown in Fig. 7e, no difference existed in TEAEs between the $100 \mathrm{mg}$ and 300 $\operatorname{mg}(\mathrm{RR}=0.94,95 \% \mathrm{CI}: 0.79 \sim 1.12, p=0.51)$.

\section{Risk of bias}

The independent risk of biases related to 4 RCTs are shown in Fig. 8. The risk for attrition bias is unclear in the studies carried out by Dodick (2019) and Lipton (2020). In addition to the measure, other studies had reported low risks of bias.

\section{Discussion}

Migraine is a prevalent neurological disease around the globe. However, previous therapies have some limitations or adverse effects, and are unresolved until now. As the importance of CGRP in the pathogenesis has been proved by the previous studies, its receptors are widely distributed in the central nervous system (CNS) and peripheral sensory neurons. Therefore, monoclonal 


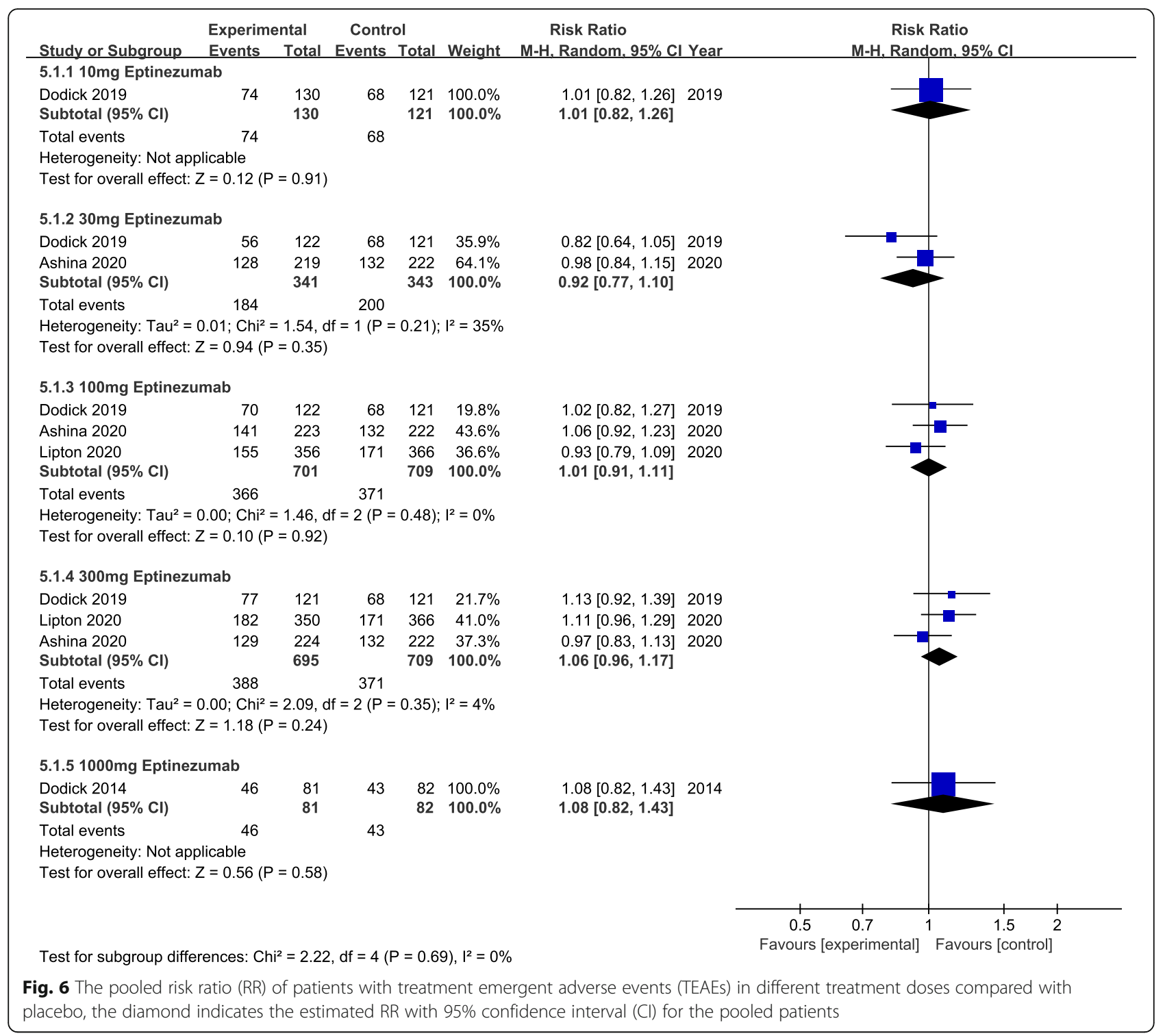

antibodies blocking the CGRP ligand or receptor have a clear advantage in the treatment strategy for episodic and chronic migraine [21].

Our study is the first meta-analysis about different dosage regimens related to the safety and efficacy of eptinezumab in the treatment for migraine, and indicated eptinezumab as excellent therapeutic agent for the migraine. Generally, a meta-analysis is a statistical method which combines the results of different researches on the similar topic and it may resolve conflicts among studies. Just as what we have done above, we gathered and analyzed the data from 4 RCTs through objective methods of meta-analysis, which enlarged the sample size and increased statistical power compared with the single available trials. So, this meta-analysis can help clinicians understand eptinezumab in clinical practice and research so that they can conduct a better clinical decision about the use of it.

During our study, we pooled 2739 participators from 4 randomized clinical trials (RCTs), which provided high clinical reliability in the research for the use of eptinezumab. Further, we gathered primary data from these articles and did not discover apparent heterogeneity in our outcomes as indicated by our statistical analysis. Subsequently, we found that eptinezumab had been divided into flexible dosage regimens in these RCTs, including $10 \mathrm{mg}, 30 \mathrm{mg}, 100 \mathrm{mg}, 300 \mathrm{mg}, 1000 \mathrm{mg}$. Further, by comparing the primary efficacy outcomes mean monthly migraine days (MMDs), baseline to 12 weeks, we proved that treatment with $30 \mathrm{mg}, 100 \mathrm{mg}, 300 \mathrm{mg}$ can cause effective reduction in monthly migraine days (MMDs) compared with placebo. Whereas, for the secondary 


\section{a

\begin{tabular}{|c|c|c|c|c|c|c|c|c|c|}
\hline \multirow[b]{2}{*}{ Study or Subgroup } & \multicolumn{3}{|c|}{ 100mg Eptinezumab } & \multicolumn{3}{|c|}{ 300mg Eptinezumab } & \multicolumn{3}{|c|}{ Std. Mean Difference } \\
\hline & Mean & SD & Total & Mean & SD & Total & Weight & IV. Random, $95 \% \mathrm{Cl}$ & Year \\
\hline Dodick 2019 & -7.6 & 6.74 & 118 & -8.2 & 6.82 & 114 & $16.8 \%$ & $0.09[-0.17,0.35]$ & 20 \\
\hline Lipton 2020 & -7.7 & 5.83 & 356 & -8.2 & 5.8 & 350 & $51.1 \%$ & $0.09[-0.06,0.23]$ & 202 \\
\hline Ashina 2020 & -3.9 & 2.88 & 221 & -4.3 & 3.05 & 222 & $32.1 \%$ & $0.13[-0.05,0.32]$ & \\
\hline Total $(95 \% \mathrm{Cl})$ & & & 695 & & & 686 & $100.0 \%$ & $0.10[-0.00,0.21]$ & \\
\hline
\end{tabular}

Heterogeneity: $\mathrm{Tau}^{2}=0.00 ; \mathrm{Chi}^{2}=0.17, \mathrm{df}=2(\mathrm{P}=0.92) ; \mathrm{I}^{2}=0 \%$

Test for overall effect: $Z=1.89(P=0.06)$

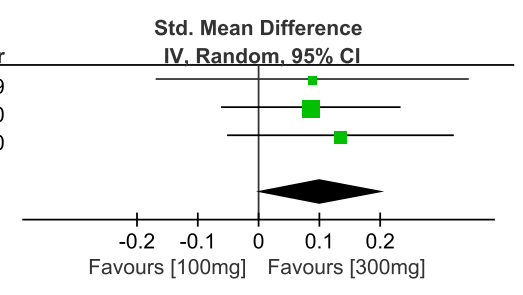

b

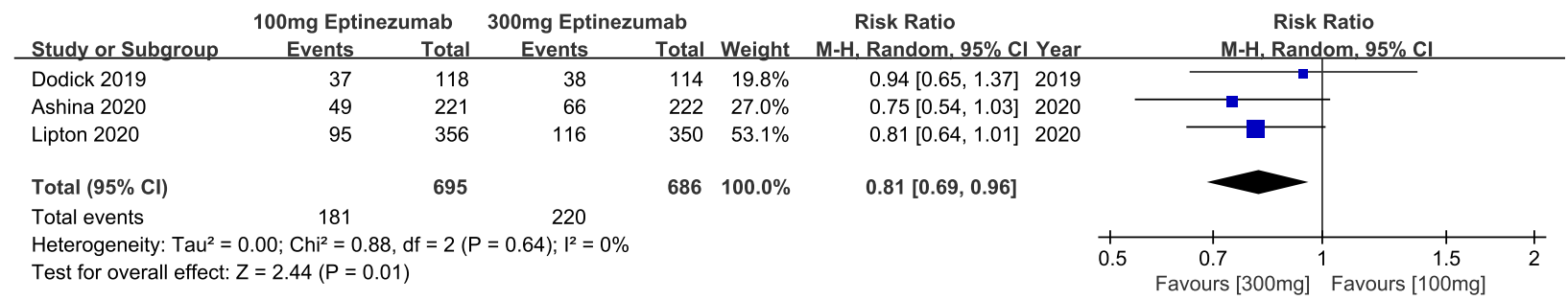

C

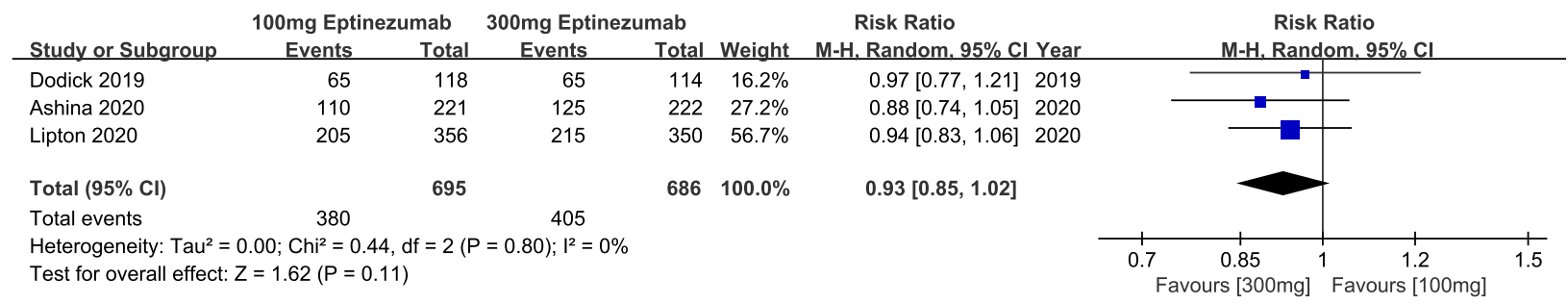

d

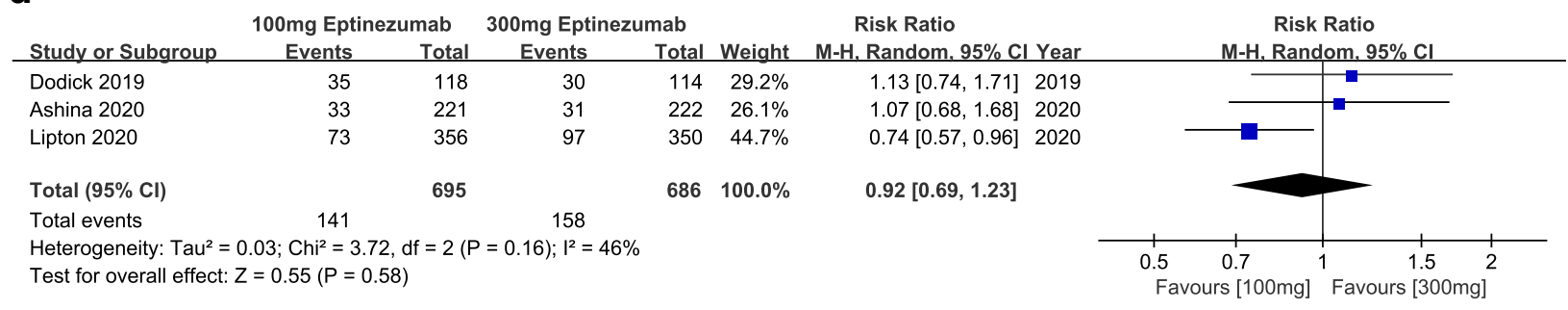

e

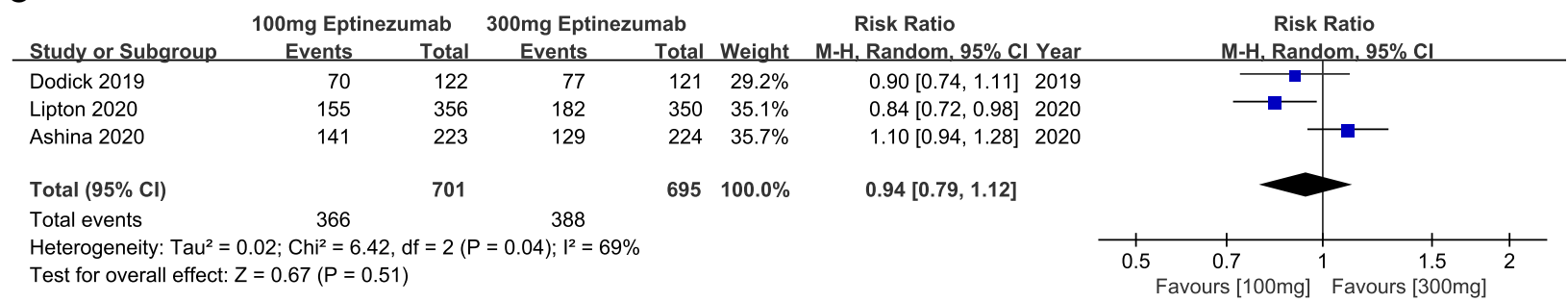

Fig. 7 Comparison of efficacy and safety outcomes between $100 \mathrm{mg}$ and $300 \mathrm{mg}$ eptinezumab. a: monthly migraine days (MMDs); b: 75\% responder rate; c: 50\% responder rate; d: patients with migraine 1 day after dosing; e: treatment emergent adverse events (TEAEs)

endpoint, all dosage regimens of eptinezumab increased the proportion of $75 \%$ responder rate except $10 \mathrm{mg}$. Similar results were observed in $50 \%$ responder rate. In addition, fewer patients suffered from migraine 1 day after $100 \mathrm{mg}$ and $300 \mathrm{mg}$ eptinezumab administration compared with $30 \mathrm{mg}$. Due to the lack of research and subsequent data, we could not continue further exploration of $10 \mathrm{mg}$ (only in the study conducted by Dodick et al. 2014) and $1000 \mathrm{mg}$ (only in the study conducted by Dodick et al. 2019) for the efficacy of eptinezumab. 

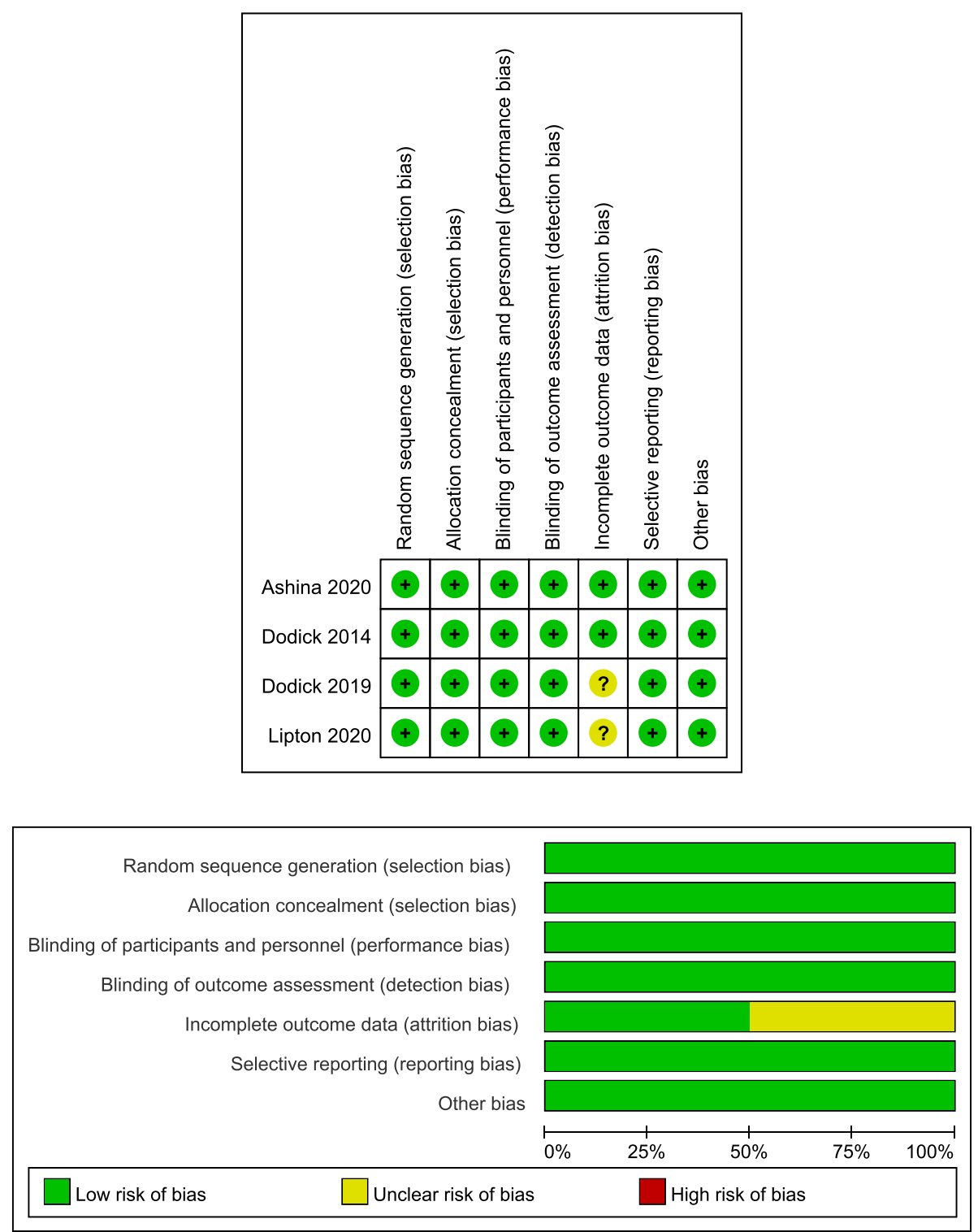

Fig. 8 Summary table for potential bias analysis for included study

However, it doesn't mean that these dosage regimens were insignificant, probably research related to it needs more time for comprehensive outcome.

By analyzing the results of different dosage regimens of eptinezumab, we found that the dosage regimens of $100 \mathrm{mg}$ and $300 \mathrm{mg}$ were more significant in the efficacy of the treatment for migraine. Moreover, the most recent phase III clinical trial conducted by Lipton et al. 2020 also employed $100 \mathrm{mg}$ and $300 \mathrm{mg}$ eptinezumab, which might confirm the tendency of exploration between the dosage regimens of $100 \mathrm{mg}$ and $300 \mathrm{mg}$ [19]. Therefore, further study was carried out for these two dosage regimens. From the perspective of the outcome related to the MMDs, baseline to 12 week, $300 \mathrm{mg}(P=$
0.06) eptinezumab showed no significant difference but potential tendency for the reduction of MMDs compared with $100 \mathrm{mg}$. Nevertheless, for the $75 \%$ responder rate, $300 \mathrm{mg}$ eptinezumab has been proved more increasing proportion than $100 \mathrm{mg}$. The result of $50 \%$ responder rate and patients with migraine 1 day after dosing couldn't indicate the difference between $100 \mathrm{mg}$ and $300 \mathrm{mg}$. To sum up, the dosage regimen of $300 \mathrm{mg}$ may have an advantage on the efficacy of the treatment for migraine. Certainly, the advantage was merely demonstrated by statistical analysis, waiting for more clinical verification.

During our study, the analysis of safety outcomesTEAEs did not indicate existence of statistical difference 
between eptinezumab and placebo $(P>0.05)$. Therefore, generally the use of eptinezumab is safe for the treatment of migraine. The result was consistent with the meta-analysis conducted by $\mathrm{Da} \mathrm{Xu}$ and Deng Chen [22] which demonstrated monoclonal antibodies blocking the CGRP ligand or receptor are safe. As reported in the previous studies, we observed that eptinezumab rarely causes serious adverse events or even death [23, 24]. Moreover, it only resulted in some mild adverse events such as upper respiratory tract infection, nausea and sinus congestion, just like the other monoclonal antibodies blocking the CGRP ligand or receptor [25]. Certainly, these studies on adverse events merely evaluated 12 weeks after the first dose. We cannot ensure whether eptinezumab will produce long lasting influence. Therefore, it still needs further comprehensive research.

After the analysis of our data, we found few limitations in our study which cannot be avoided through existing researches. Firstly, numerous previous studies have concluded evidence to use other CGRP monoclonal antibodies such as ubrogepant, galcanezumab and rimegepant, for the treatment of migraine [26-28]. However, as interventions in our study were related to different dosage regimens of eptinezumab and placebo, we can only conclude the advantages of eptinezumab compared with placebo. Probably, our study needs more horizontal comparison of eptinezumab with other CGRP monoclonal antibodies in the future. Secondly, considering different dosage regimens in 4 RCTs, $1000 \mathrm{mg}$ eptinezumab merely conducted by Dodick 2014, whereas, $10 \mathrm{mg}$ merely conducted by Dodick 2019. Moreover, part of statistics from Dodick 2019, Ashina 2020 and Lipton 2020 did not indicate standard deviation (SD) clearly. However, in the present study, we ultimately achieved SD using statistical algorithm on our own. Therefore, the accuracy of the results needs further verification. Thirdly, we combined studies about episodic migraine with those about chronic migraine which may increase the heterogeneity of comparisons. Fourth, this meta-analysis was not registered prior to data collection. Except for the limitation above, we also cannot ignore the lack of adherence in the therapy of migraine which occurred in our 4 RCTs in a way. This also encountered by few traditional treatments for migraine $[29,30]$.

\section{Conclusion}

In conclusion, eptinezumab showed outstanding efficacy for the treatment of migraine, especially dosage regimen of $300 \mathrm{mg}$. Meanwhile, no apparent differences existed when compared with placebo from the perspective of safety. Nonetheless, we are looking forward for more studies related to the eptinezumab so that it may have a promising future in the therapy strategy of migraine.

\section{Abbreviations}

CGRP: Calcitonin gene-related peptide; RCTs: Randomized controlled trials; RR: Risk ratio; SMD: Standard mean difference; MMDs: Monthly migraine day; TEAEs: Treatment emergent adverse events; Cl: Confidence interval;

CNS: Central nervous system

\section{Supplementary Information}

The online version contains supplementary material available at https://doi. org/10.1186/s10194-021-01220-y.

Additional file 1: Table S1: Inclusion, exclusion criteria, outcome assessments, conclusions and data acquisition time of the included studies.

\section{Acknowledgements}

Not applicable.

Code availability

Not applicable.

Consent to participate

Not applicable.

Authors' contributions

ZW and ZC were the principal investigators. ZYY and TX designed the study and developed the analysis plan; SJC analyzed the data and performed the meta-analysis; GJL and XYY contributed to the writing of the article. XW and $\mathrm{SG}$ revised the manuscript and polished the language. All authors read and approved the final submitted paper.

Funding

This work was supported by the Suzhou Health Talents Training Project (GSWS2019002).

\section{Availability of data and materials}

All data generated or analyzed during this study are included in this published article and its supplementary information files.

\section{Declarations}

Ethics approval

Not applicable.

\section{Consent for publication}

Not applicable.

\section{Competing interests}

The authors declare that they have no competing interests.

\section{Author details}

'Department of Neurosurgery \& Brain and Nerve Research Laboratory, The First Affiliated Hospital of Soochow University, 188 Shizi Street, Suzhou 215006, Jiangsu Province, China. ${ }^{2}$ Department of Neurology, The First Affiliated Hospital of Soochow University, Suzhou 215006, Jiangsu Province, China. ${ }^{3}$ Department of Neurosurgery, The People's Hospital of SND, Suzhou 215129, Jiangsu Province, China.

Received: 4 November 2020 Accepted: 22 February 2021

Published online: 06 March 2021

\section{References}

1. Schwedt TJ (2014) Chronic migraine. BMJ 348:g1416

2. Headache Classification Committee of the International Headache, S (2013) The International Classification of Headache Disorders, 3rd edition (beta version). Cephalalgia 33(9):629-808

3. Stewart WF et al (2010) Employment and work impact of chronic migraine and episodic migraine. J Occup Environ Med 52(1):8-14 
4. Collaborators, G.B.D.H (2018) Global, regional, and national burden of migraine and tension-type headache, 1990-2016: a systematic analysis for the Global Burden of Disease Study 2016. Lancet Neurol 17(11):954-976

5. Lipton, R.B. and S.D. Silberstein, Episodic and chronic migraine headache: breaking down barriers to optimal treatment and prevention. Headache, 2015. 55 Suppl 2: p. 103-122; quiz 123-6

6. Lambru $\mathrm{G}$ et al (2018) Emerging drugs for migraine treatment: an update. Expert Opin Emerg Drugs 23(4):301-318

7. Cameron C et al (2015) Triptans in the acute treatment of migraine: a systematic review and network meta-analysis. Headache 55(Suppl 4):221235

8. Dodick DW et al (2019) Ubrogepant for the treatment of migraine. N Engl J Med 381(23):2230-2241

9. Goadsby PJ et al (2019) Phase 3 randomized, placebo-controlled, doubleblind study of lasmiditan for acute treatment of migraine. Brain 142(7): 1894-1904

10. Tepper SJ (2018) History and review of anti-calcitonin gene-related peptide (CGRP) therapies: from translational research to treatment. Headache 58(Suppl 3):238-275

11. Diener HC (2014) CGRP as a new target in prevention and treatment of migraine. Lancet Neurol 13(11):1065-1067

12. Peters GL (2019) Migraine overview and summary of current and emerging treatment options. Am J Manag Care 25(2 Suppl):S23-S34

13. Scuteri $D$ et al (2019) Eptinezumab for the treatment of migraine. Drugs Today (Barc) 55(11):695-703

14. Sacco $S$ et al (2019) European headache federation guideline on the use of monoclonal antibodies acting on the calcitonin gene related peptide or its receptor for migraine prevention. J Headache Pain 20(1):6

15. Tiseo C et al (2019) How to integrate monoclonal antibodies targeting the calcitonin gene-related peptide or its receptor in daily clinical practice. J Headache Pain 20(1):49

16. Ashina $\mathrm{M}$ et al (2020) Eptinezumab in episodic migraine: a randomized, double-blind, placebo-controlled study (PROMISE-1). Cephalalgia 40(3):241254

17. Dodick DW et al (2014) Safety and efficacy of ALD403, an antibody to calcitonin gene-related peptide, for the prevention of frequent episodic migraine: a randomised, double-blind, placebo-controlled, exploratory phase 2 trial. Lancet Neurol 13(11):1100-1107

18. Smith JT et al (2016) Randomized, double-blind, placebo-controlled trial of ALD403, an anti-CGRP peptide antibody in the prevention of chronic migraine. Headache 56(8):1391

19. Lipton RB et al (2020) Efficacy and safety of eptinezumab in patients with chronic migraine: PROMISE-2. Neurology 94(13):E1365-E1377

20. Liberati A et al (2009) The PRISMA statement for reporting systematic reviews and meta-analyses of studies that evaluate healthcare interventions: explanation and elaboration. BMJ 339:b2700

21. Goadsby PJ et al (2017) Pathophysiology of migraine: a disorder of sensory processing. Physiol Rev 97(2):553-622

22. Xu D et al (2019) Safety and tolerability of calcitonin-gene-related peptide binding monoclonal antibodies for the prevention of episodic migraine meta-analysis of randomized controlled trials. Cephalalgia 39(9):1164-1179

23. Allan B et al (2019) Prevail: an open-label phase 3 trial to evaluate the safety of eptinezumab administered intravenously in patients with chronic migraine. Headache 59:105

24. Diener HC (2020) Eptinezumab for the prevention of episodic migraine. Arzneimitteltherapie 38(6):250-251

25. Zhu Y et al (2018) The efficacy and safety of calcitonin gene-related peptide monoclonal antibody for episodic migraine: a meta-analysis. Neurol Sci 39(12):2097-2106

26. Yang $Y$ et al (2020) Safety and efficacy of Ubrogepant for the acute treatment of episodic migraine: a meta-analysis of randomized clinical trials. CNS Drugs 34(5):463-471

27. Gao B et al (2019) Efficacy and safety of Rimegepant for the acute treatment of migraine: evidence from randomized controlled trials. Front Pharmacol 10:1577

28. Yang Y et al (2020) Different doses of galcanezumab versus placebo in patients with migraine and cluster headache: a meta-analysis of randomized controlled trials. J Headache Pain 21(1):14

29. Berger A et al (2012) Adherence with migraine prophylaxis in clinical practice. Pain Pract 12(7):541-549
30. Hepp Z et al (2017) Persistence and switching patterns of oral migraine prophylactic medications among patients with chronic migraine: a retrospective claims analysis. Cephalalgia 37(5):470-485

\section{Publisher's Note}

Springer Nature remains neutral with regard to jurisdictional claims in published maps and institutional affiliations.
Ready to submit your research? Choose BMC and benefit from:

- fast, convenient online submission

- thorough peer review by experienced researchers in your field

- rapid publication on acceptance

- support for research data, including large and complex data types

- gold Open Access which fosters wider collaboration and increased citations

- maximum visibility for your research: over $100 \mathrm{M}$ website views per year

At $\mathrm{BMC}$, research is always in progress.

Learn more biomedcentral.com/submissions 